\title{
Mesure de la déformation d'un matériau piézoélectrique par interférométrie
}

\author{
G. Feld, E. Labouré, E. Hoang, B. Revol \\ feld@eea.ens-cachan.fr, laboure@satie.ens-cachan.fr, hoang@satie.ens-cachan.fr, revol@satie.ens-cachan.fr \\ École normale supérieure de Cachan, 61 avenue du Président Wilson, 94235 Cachan
}

\begin{abstract}
RÉSUMÉ : Cet article présente une manipulation de physique appliquée concernant la caractérisation par interférométrie d'un matériau piézoélectrique. Cette manipulation s'insère dans une unité d'enseignement de la troisième année de la licence de physique, parcours IST (Information, Systèmes et Technologie - EEA). L'objectif de cette unité, intitulée Matériaux pour l'information et l'énergie, est de faire le lien entre la physique des matériaux et le génie électrique. Au cours de cette séance de manipulation, les étudiants étudient successivement les interférences lumineuses, la mesure de déformation par interférométrie et enfin l'application à la mesure d'un coefficient piézoélectrique. Cette manipulation de physique appliquée permet d'aborder différents domaines de la physique : les matériaux, l'optique ainsi que la métrologie. L'expérience nous a montrés que l'on pouvait aborder ce sujet avec simplement quelques connaissances en optique géométrique.
\end{abstract}

Mots clés : matériaux électroactifs, piézoélectricité, interférences lumineuses, mesures par interférométrie.

\section{INTRODUCTION}

Les nouveaux matériaux électroactifs possèdent des propriétés de couplage électromécanique intéressantes qui permettent d'innover en matière de conversion électromécanique. Avant d'aborder l'étude de ces nouveaux actionneurs, nous proposons aux étudiants une unité d'enseignement traitant des matériaux pour le génie électrique. Des séances de manipulation permettent d'illustrer ce cours, l'une d'elles traite des matériaux piézoélectriques. Au cours de cette séance, d'une durée de quatre heures, deux sujets sont abordés. Le premier et le seul présenté dans cet article concerne la mesure par interférométrie d'un des paramètres d'une céramique piézoélectrique. Quant au deuxième, il traite de la déformation d'une poutre sur laquelle deux plaquettes de matériau piézoélectrique ont été collées.

Un rappel des équations de la piézoélectricité est tout d'abord donné dans la section 2 puis les conditions d'obtention d'une figure d'interférences en lumière monochromatique sont rappelées dans la section 3 . L'application de l'interférométrie à la mesure d'un des coefficients piézoélectriques est présentée dans la section 4.

\section{LA PIÉZOÉLECTRICITE [1]}

L'effet piézoélectrique traduit le couplage entre les effets mécaniques et les effets électriques. C'est aux frères Curie que l'on attribue la découverte de l'effet direct en 1880. Certains matériaux ont la propriété de se polariser sous l'effet d'une contrainte mécanique (figure 1-a), c'est l'effet piézoélectrique direct. Cette polarisation change de signe avec la contrainte. L'effet piézoélectrique est réversible et ainsi, ces matériaux se déforment sous l'effet d'une polarisation électrique résultant de l'application d'un champ électrique (figure 1-b), c'est l'effet inverse.

Les milieux piézoélectriques sont, par définition, anisotropes. Afin d'étudier leurs propriétés, il est donc nécessaire de définir précisément les directions du repère d'étude. On choisit en général l'axe 3 selon la direction du champ de polarisation rémanente $\overrightarrow{P_{0}}$. Les axes 1,2 et 3 forment un trièdre direct (figure 2). Les chiffres 4,5 et 6 permettent de définir les indices relatifs aux contraintes de cisaillement autour des axes 1,2 et 3 .

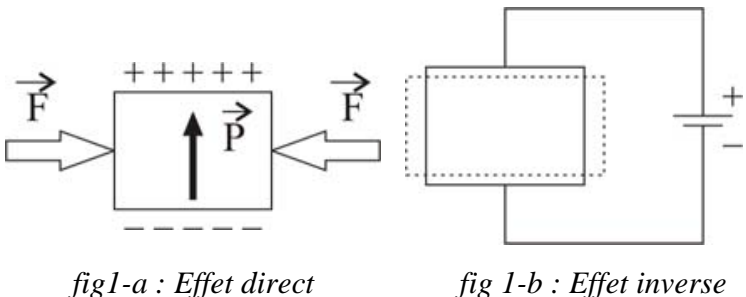

fig1 : Effet piézoélectrique

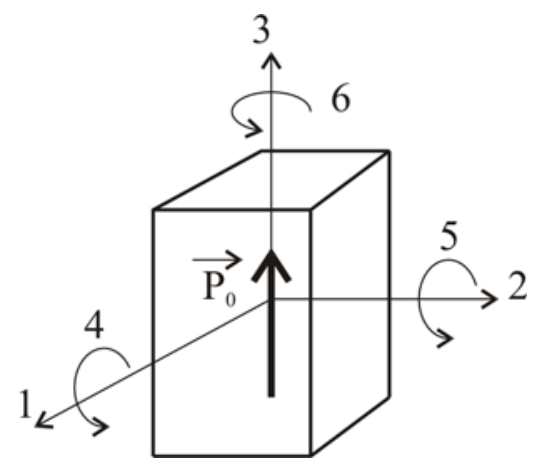

fig 2 : Axes de référence

\section{1 Équations de la piézoélectricité}

Les équations de la piézoélectricité relient les variables électriques (induction et champ électrique) aux variables mécaniques (déformation relative et contrainte). 


\section{Définition des grandeurs}

Les différentes grandeurs physiques et notations associées sont données dans le tableau ci-dessous.

\begin{tabular}{|l|c|l|c|l|}
\hline nature & $\begin{array}{c}\text { nota- } \\
\text { tions }\end{array}$ & & unité & dim \\
\hline \multirow{5}{*}{ électrique } & $\mathbf{D}$ & induction électrique & $\mathrm{C} / \mathrm{m}^{2}$ & $3 \times 1$ \\
& $\mathbf{E}$ & champ électrique & $\mathrm{V} / \mathrm{m}$ & $3 \times 1$ \\
& $\varepsilon$ & permittivité & $\mathrm{F} / \mathrm{m}$ & $3 \times 3$ \\
& $\beta$ & inverse de la permittivité & $\mathrm{m} / \mathrm{F}$ & $3 \times 3$ \\
\hline \multirow{3}{*}{ mécanique } & $\mathrm{S}$ & tenseur de déformation & & $6 \times 1$ \\
& $\mathrm{~T}$ & tenseur des contraintes & $\mathrm{N} / \mathrm{m}^{2}$ & $6 \times 1$ \\
& $\mathrm{c}$ & rigidité & $\mathrm{N} / \mathrm{m}^{2}$ & $6 \times 6$ \\
& $\mathrm{~S}$ & souplesse & $\mathrm{m} / \mathrm{N}$ & $6 \times 6$ \\
\hline \multirow{3}{*}{ piézo- } & $\mathrm{h}$ & constantes piézoélectri- & $\mathrm{N} / \mathrm{C}$ & $3 \times 6$ \\
-électrique & $\mathrm{e}$ & ques du matériau & $\mathrm{N} / \mathrm{Vm}$ & $3 \times 6$ \\
& $\mathrm{~g}$ & & $\mathrm{~m} / \mathrm{C}$ & $3 \times 6$ \\
& $\mathrm{~d}$ & & $\mathrm{~m} / \mathrm{V}$ & $3 \times 6$ \\
\hline
\end{tabular}

tableau 1 : Définition des grandeurs

\section{Équations}

Les équations de la piézoélectricité sont rappelées dans le tableau 2 [1], où ()$^{t}$ indique la transposée de la matrice considérée et ()$^{\mathrm{X}}$ précise que la grandeur considérée est définie à $\mathrm{X}$ constant.

\begin{tabular}{|l|l|l|}
\hline & \multicolumn{1}{|c|}{$\mathbf{T}$} & \multicolumn{1}{c|}{$\mathbf{S}$} \\
\hline \multirow{2}{*}{$\mathbf{E}$} & $\begin{array}{l}\mathbf{S}=\mathrm{s}^{\mathrm{E}} \cdot \mathbf{T}+\mathrm{d}^{\mathrm{t}} . \mathbf{E} \\
\mathbf{D}=\mathrm{d} \cdot \mathbf{T}+\varepsilon^{\mathrm{T}} . \mathbf{E}\end{array}$ & $\mathbf{T}=\mathrm{c}^{\mathrm{E}} . \mathbf{S}-\mathrm{e}^{\mathrm{t}} . \mathbf{E}$ \\
& $\mathbf{D}=\mathrm{e} \cdot \mathbf{S}+\varepsilon^{\mathrm{S}} . \mathbf{E}$ \\
\hline \multirow{2}{*}{$\mathbf{D}$} & $\begin{array}{l}\mathbf{S}=\mathrm{s}^{\mathrm{D}} \cdot \mathbf{T}+\mathrm{g}^{\mathrm{t}} . \mathbf{D} \\
\mathbf{E}=-\mathrm{g} \cdot \mathbf{T}+\beta^{\mathrm{t}} . \mathbf{D}\end{array}$ & $\mathbf{T}=\mathrm{c}^{\mathrm{D}} . \mathbf{S}-\mathrm{h}^{\mathrm{t}} . \mathbf{D}$ \\
& $\mathbf{E}=-\mathrm{h} \cdot \mathbf{S}+\beta^{\mathrm{S}} . \mathbf{E}$ \\
\hline
\end{tabular}

tableau 2 : Équations de la piézoélectricité

\section{Mode de déformation}

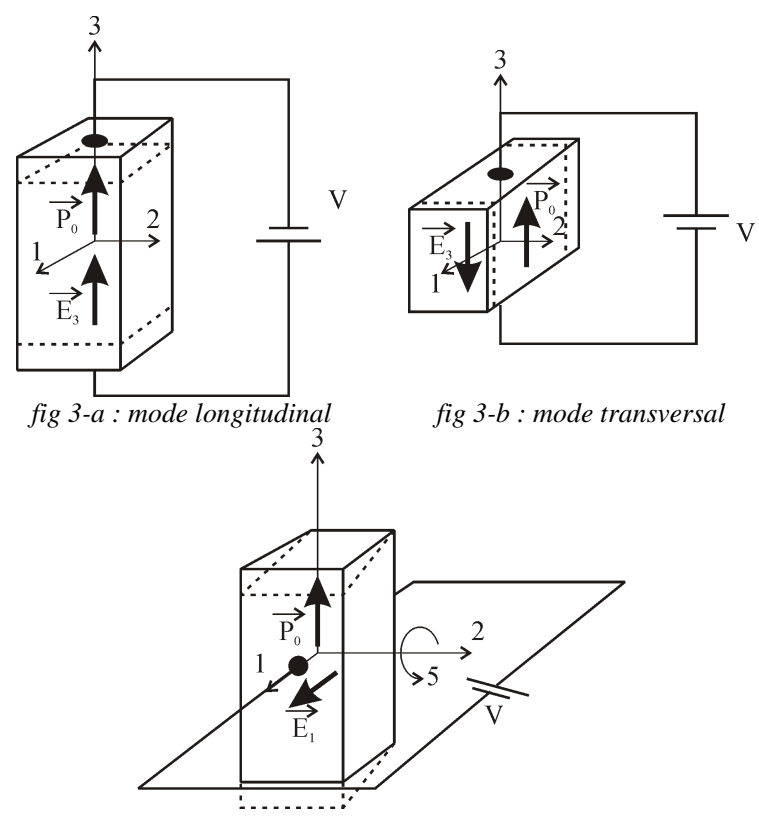

fig 3-c : mode de cisaillement

fig 3 : Les différents modes de déformation

On distingue trois modes principaux de déformation d'un échantillon piézoélectrique : le mode longitu- dinal (33), le mode transversal (31) et le mode de cisaillement (51) qui sont illustrés sur la figure 3. Les déformations selon les axes perpendiculaires à la grande longueur n'ont pas été représentées sur les figures 3-a et 3-b.

Le matériau utilisé dans la manipulation travaillant dans le mode longitudinal, nous allons dans la suite nous intéresser plus particulièrement à ce mode de déformation.

\section{2 Équations du mode longitudinal}

En considérant les symétries des cristaux de la céramique piézoélectrique et en supposant qu'il n'existe pas de contrainte mécanique selon les axes 1 et 2 , les équations de ce mode s'écrivent

et

$$
\mathrm{S}_{3}=\mathrm{s}_{33}^{\mathrm{E}} \cdot \mathrm{T}_{3}+\mathrm{d}_{33} \cdot \mathrm{E}_{3}
$$

$$
\mathrm{D}_{3}=\mathrm{d}_{33} \cdot \mathrm{T}_{3}+\varepsilon_{33}^{\mathrm{T}} \cdot \mathrm{E}_{3} .
$$

En l'absence de contrainte selon l'axe 3 (le barreau est libre de se déplacer), la première équation se simplifie et devient

$$
\mathrm{S}_{3}=\mathrm{d}_{33} \cdot \mathrm{E}_{3} .
$$

La déformation relative $S_{3}$ s'exprime en fonction de l'allongement par la relation

$$
\mathrm{S}_{3}=\frac{\Delta \mathrm{l}}{1} .
$$

Or, le champ électrique est relié à la différence de potentiel V par l'expression

$$
\mathrm{E}_{3}=\frac{\mathrm{V}}{1} .
$$

Ainsi, on en déduit la relation suivante

$$
\Delta \mathrm{l}=\mathrm{d}_{33} . \mathrm{V} .
$$

Les grandeurs I, $\Delta \mathrm{l}$ et $\mathrm{V}$ sont définies sur la figure 4 .

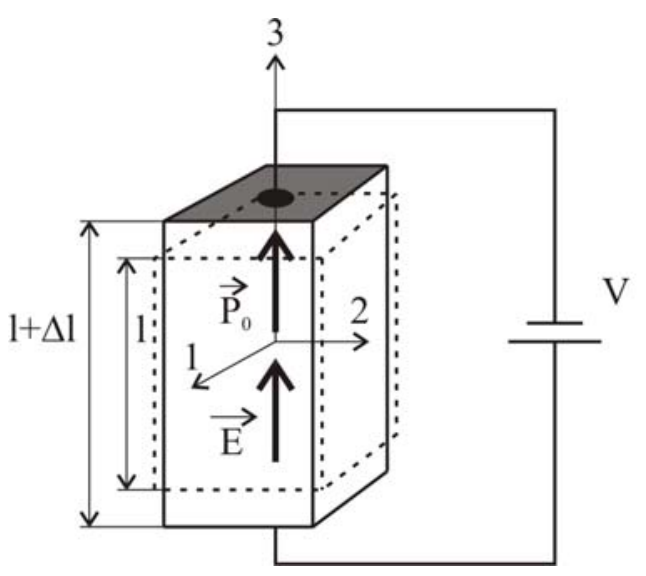

fig 4 : Mode longitudinal

\subsection{Transducteur multicouches}

Pour limiter, à déplacement donné, les niveaux de tension appliqués, des céramiques sont empilées têtebêche par rapport à leur polarisation (figure 5). Les différentes pastilles sont connectées en parallèle. L'actionneur utilisé, de hauteur totale égale à $18 \mathrm{~mm}$, 
est constitué de 280 couches d'épaisseur $60 \mu \mathrm{m}$. Le matériau est une céramique PZT (Plomb, Zirconium et Titane) de référence PIC255 [3]. En l'absence de contrainte, le déplacement à vide s'écrit :

$$
\Delta \mathrm{l}=\mathrm{n} \cdot \mathrm{d}_{33} . \mathrm{V}
$$

où $\mathrm{n}$ est le nombre de couches.
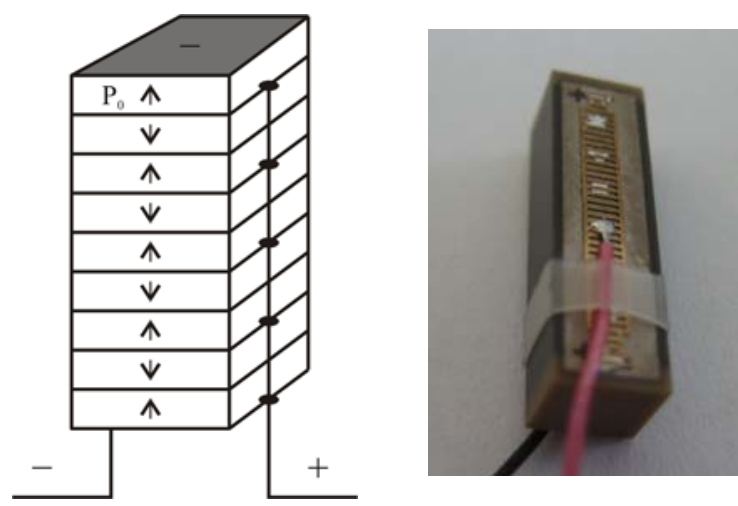

fig 5 : Transducteur multicouches

Cet empilement permet d'obtenir des déplacements d'une dizaine de micromètres pour une tension inférieure à une centaine de Volts, tout en développant des forces maximales de plusieurs centaines de Newton en régime d'encastrement. Par contre, la capacité élevée du condensateur formée par l'empilement limitera la fréquence de la tension d'alimentation, et les vitesses d'entraînement seront au maximum de $1 \mathrm{~mm} / \mathrm{s}$. Une vanne de carburant dans le domaine aéronautique est motorisée par ce type d'actionneur [2].

\section{INTERFÉROMÉTRIE}

La mesure de déplacement ou de déformation de quelques micromètres fait souvent appel à des mesures interférométriques réalisées en lumière monochromatique de longueur d'onde $\lambda$ [4]. Cette méthode consiste à comparer les déplacements à la période spatiale des ondes. Utilisée industriellement dans le contrôle non destructif, elle permet de réaliser des mesures très précises sans contact mécanique. Ce principe est, entre autre, utilisé pour étudier le comportement des structures aéronautiques soumises à des contraintes mécaniques. Ce type de mesures est parfaitement adapté à la détermination de la déformation d'une céramique piézoélectrique qui est typiquement de l'ordre de la centaine de nanomètres.

Pour décrire correctement ces interférences, il est nécessaire de prendre en compte le caractère ondulatoire de la lumière qui sera assimilable à une onde électromagnétique. Quant au récepteur, ici l'œil, il est sensible à l'éclairement qui est proportionnel au carré de l'amplitude du champ électrique. En un point donné de l'espace, le champ électrique s'écrit

$$
\vec{E}=E \cos (\omega \cdot \mathrm{t}-\varphi) \cdot \overrightarrow{\mathrm{u}},
$$

où $\overrightarrow{\mathrm{u}}$ est un vecteur unitaire. L'œil est sensible à l'éclairement noté I.

$$
\mathrm{I}=\alpha \cdot \mathrm{E}^{2} .
$$

Les interférences se produisent en un point de l'espace lorsque les composantes de deux champs de même fréquence se superposent.

\subsection{Composition de deux vibrations}

Considérons deux ondes de même pulsation et parallèles entre elles (rayons lumineux peu inclinés selon l'axe de propagation) s'écrivant au point $M$

$$
\overrightarrow{\mathrm{E}}_{1 \mathrm{M}}=\mathrm{E}_{1 \mathrm{M}} \cos \left(\omega \cdot \mathrm{t}-\varphi_{1 \mathrm{M}}\right) \cdot \overrightarrow{\mathrm{u}}_{\mathrm{M}}
$$

et

$$
\overrightarrow{\mathrm{E}}_{2 \mathrm{M}}=\mathrm{E}_{2 \mathrm{M}} \cos \left(\omega \cdot \mathrm{t}-\varphi_{2 \mathrm{M}}\right) \cdot \overrightarrow{\mathrm{u}}_{\mathrm{M}},
$$

où $\overrightarrow{\mathrm{u}}_{\mathrm{M}}$ est un vecteur unitaire.

L'éclairement résultant au point $\mathrm{M}$ est

$$
I_{M}=\alpha\left(E_{1 M}^{2}+E_{2 M}^{2}+2 \cdot E_{1 M} \cdot E_{2 M} \cdot \cos \left(\varphi_{2 M}-\varphi_{1 M}\right)\right) .
$$

On en déduit que, dans le cas très fréquent où les deux vibrations ont une même amplitude indépendante du point $\mathrm{M}$, la relation précédente s'écrit

$$
\mathrm{I}_{\mathrm{M}}=2 \cdot \alpha \cdot \mathrm{E}^{2}\left(1+\cos \left(\varphi_{2 \mathrm{M}}-\varphi_{1 \mathrm{M}}\right)\right) .
$$

\subsection{Conditions d'observation des figures d'interférences}

En général le terme $\cos \left(\varphi_{2 M}-\varphi_{1 M}\right)$ évolue au cours du temps et sa valeur moyenne sur le durée d'observation est nulle, si bien que l'éclairement apparaît uniforme de valeur

$$
\mathrm{I}=2 . \alpha \cdot \mathrm{E}^{2} \text {. }
$$

En pratique, pour observer une modulation spatiale de l'éclairement, il est nécessaire de faire interférer des ondes issues d'une même source (vibration cohérente) mais ayant suivi des chemins différents. Dans ces conditions, le terme $\varphi_{2 \mathrm{M}}-\varphi_{1 \mathrm{M}}$ ne dépendra que des trajets géométriques.

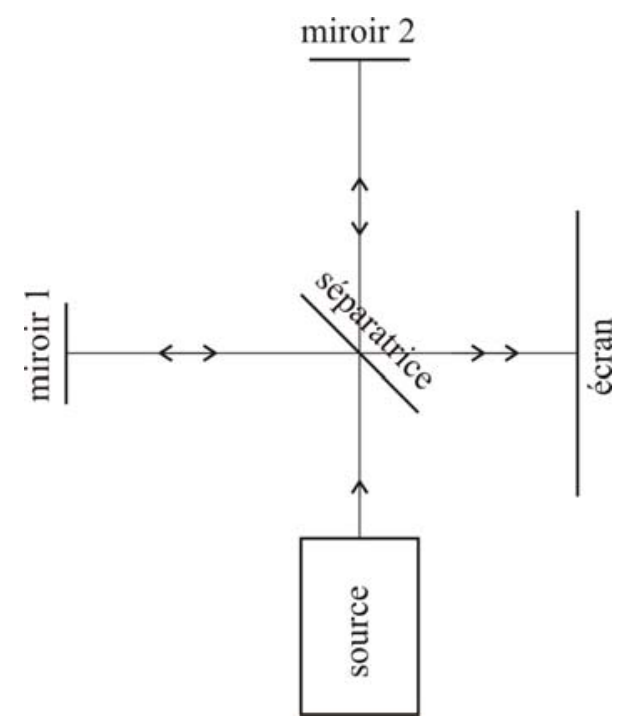

fig 6 : Interféromètre de Michelson

Un des dispositifs couramment utilisé est l'interféromètre de Michelson dont le schéma de principe est donné sur la figure 6 . Une onde issue d'une 
source est « séparée en deux » par une lame semi réfléchissante (séparatrice). L'onde réfléchie sur la séparatrice (miroir 1) et l'onde transmise (miroir 2) peuvent alors interférer et, sous certaines conditions, former sur l'écran une figure d'interférences.

\subsection{Détermination de la différence de marche}

Les lois de Snell-Descartes montrent que l'on peut ramener l'étude de la source $S$, de la séparatrice et du miroir 1 à celle d'une source ponctuelle $S_{1}$. La figure 7 montre la construction permettant de déterminer la position de la source $\mathrm{S}_{1}$. On commence par déterminer l'image de $\mathrm{S}\left(\mathrm{S}_{1}{ }_{1}\right)$ par la séparatrice et ensuite l'image de $\mathrm{S}_{1}$ par le miroir.

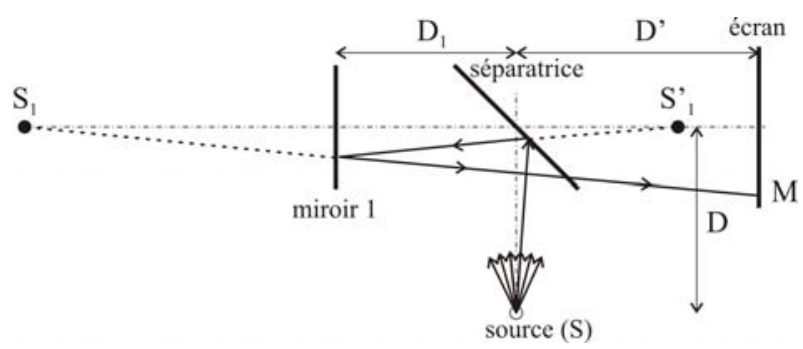

fig 7 : Trajet lumineux de l'onde réfléchie

De la même façon, la source $\mathrm{S}$, le miroir 2 et la séparatrice sont équivalents à une source ponctuelle $\mathrm{S}_{2}$.

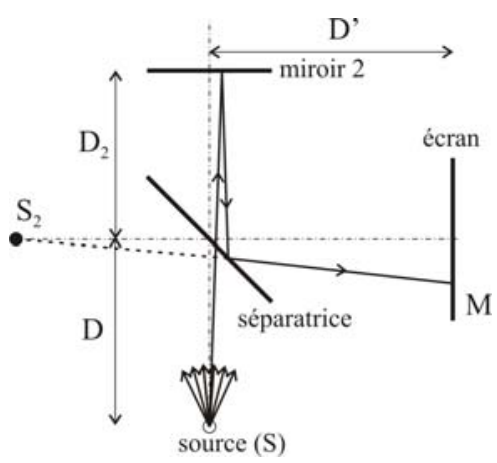

fig 8 : Trajet lumineux de l'onde transmise

On en déduit que, dans le cas d'une source ponctuelle, l'interféromètre de Michelson est équivalent au schéma de la figure 9 où les deux sources $S_{1}$ et $S_{2}$ sont cohérentes,

$$
\mathrm{D}_{\text {léq }}=2 \cdot \mathrm{D}_{1}+\mathrm{D}+\mathrm{D}^{\prime}
$$

et

$$
\mathrm{D}_{\text {2éq }}=2 . \mathrm{D}_{2}+\mathrm{D}+\mathrm{D}^{\prime} \text {, }
$$

où $\mathrm{D}_{1}, \mathrm{D}_{2}, \mathrm{D}$ et $\mathrm{D}$ ' sont définis sur les figures 7 et 8 .

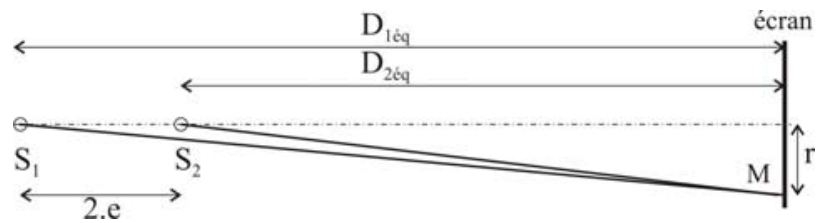

fig 9 : Sources équivalentes

La différence de marche au point $\mathrm{M}$ entre les deux ondes issues des deux sources s'écrit

$$
\delta_{\mathrm{M}}=\mathrm{S}_{1} \mathrm{M}-\mathrm{S}_{2} \mathrm{M} .
$$

L'éclairement au point $\mathrm{M}$ est donné par la relation

$$
\mathrm{I}_{\mathrm{M}}=2 \cdot \alpha \cdot \mathrm{E}^{2}\left(1+\cos \left(\frac{2 \cdot \pi \cdot \delta_{\mathrm{M}}}{\lambda}\right)\right) .
$$

Soient

$$
\mathrm{D}_{\mathrm{m}}=\frac{\mathrm{D}_{1 \mathrm{eq}}+\mathrm{D}_{2 \mathrm{eq}}}{2}
$$

la distance moyenne et

$$
\mathrm{e}=\frac{\mathrm{D}_{1 \mathrm{eq}}-\mathrm{D}_{2 \mathrm{eq}}}{2}=\mathrm{D}_{1}-\mathrm{D}_{2} \text {. }
$$

En supposant que $D_{m}>>e$, la différence de marche s'écrit

$$
\delta_{\mathrm{M}} \approx 2 \cdot \mathrm{e}\left(1-\frac{\mathrm{r}^{2}}{2 \cdot \mathrm{D}_{\mathrm{m}}^{2}}\right) .
$$

L'éclairement sur l'écran est donc constant sur des cercles de rayon $r$. La figure d'interférences obtenue sur l'écran est formée d'anneaux. Un exemple, obtenu sur le banc de mesures, est représenté sur la figure 10 .

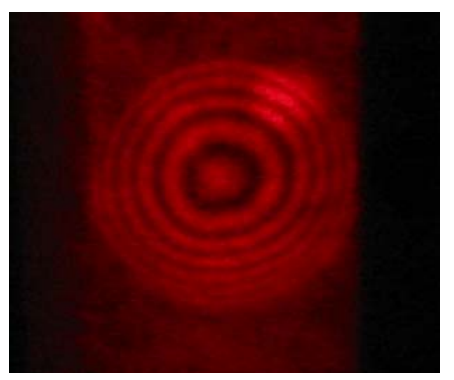

fig 10 : Figure d'interférences

\section{MESURE DE LA DÉFORMATION D'UNE CÉRAMIQUE PIÉZOÉLECTRIQUE PAR IN- TERFÉROMETRIE}

Afin de mesurer l'allongement de la céramique par méthode interférométrique, le miroir 2 a été collé sur le matériau à caractériser (figure 11).

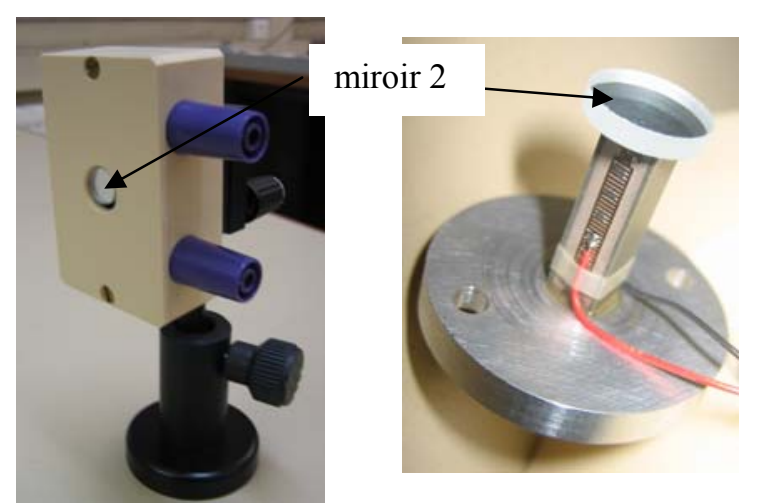

fig 11 : Céramique piézoélectrique à caractériser

Une alimentation permet d'appliquer une tension comprise entre 0 et $100 \mathrm{~V}$ sur la céramique (figures 12 et 13).Une photodiode, associée à un amplificateur de transconductance, permet d'avoir une tension image de 
l'éclairement au centre de la figure d'interférences où l'éclairement s'écrit

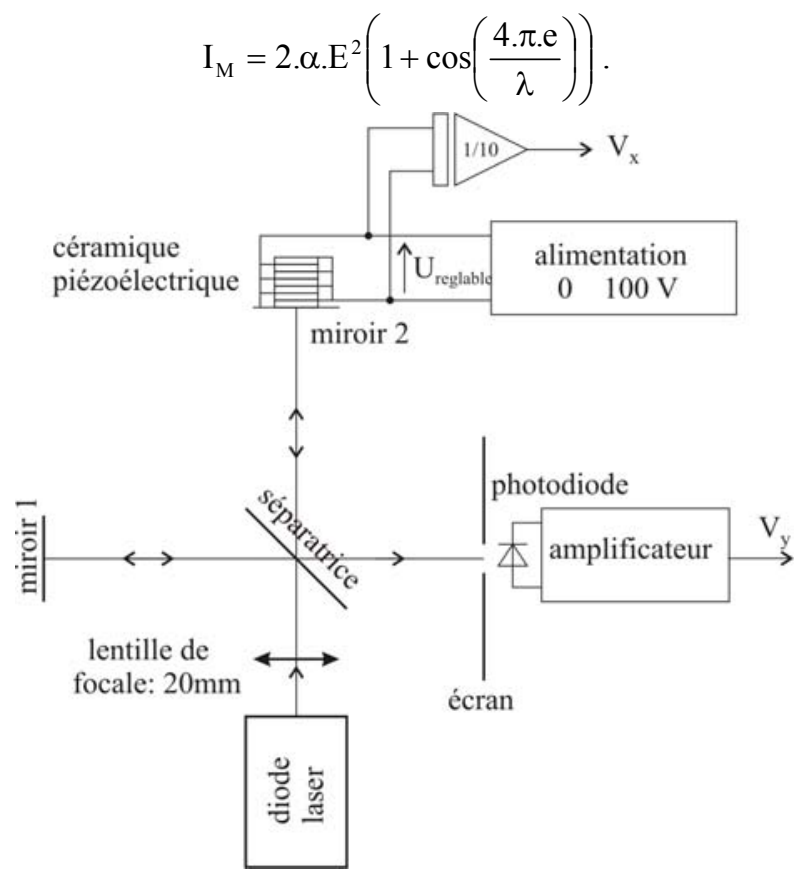

fig 12 : Synoptique du banc de mesures

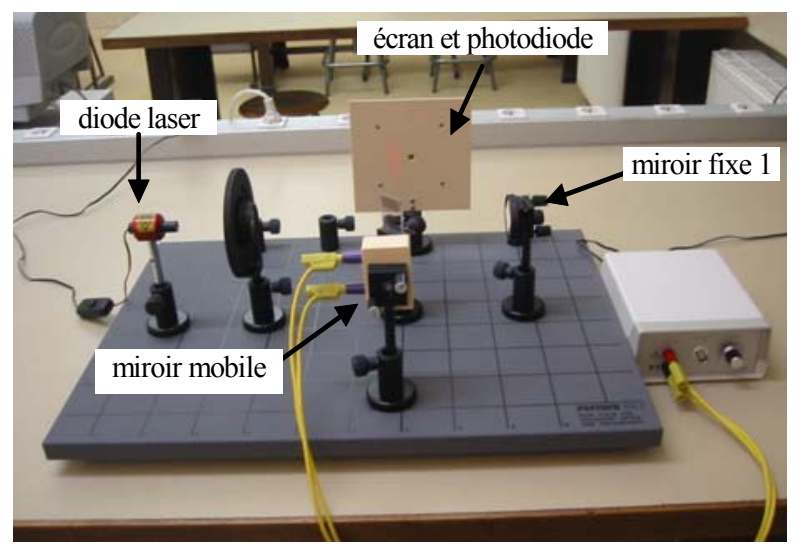

fig 13 : Photographie de l'interféromètre

Lorsque la tension appliquée sur la céramique augmente, le miroir 2 se déplace et la figure d'interférences est modifiée et le centre de la figure passe du sombre au brillant. Entre deux centres brillants consécutifs, la céramique piézoélectrique s'est déformée d'une distance

$$
\Delta \mathrm{l}=\frac{\lambda}{2} .
$$

De cet enregistrement, nous pouvons en déduire le coefficient piézoélectrique $d_{33}$ :

$$
\mathrm{d}_{33}=\frac{\Delta \mathrm{l}}{\mathrm{n} \cdot \Delta \mathrm{V}}
$$

où $\delta 1$ correspond à l'allongement de la céramique lorsque la tension a varié de $\Delta \mathrm{V}$. La variation de tension $\Delta \mathrm{V}$ de la figure 14 correspond à la succession de quatre centres brillants, soit un déplacement relatif du miroir de $\Delta \mathrm{l}=2 . \lambda$, d'où

$$
\mathrm{d}_{33}=\frac{2 . \lambda}{\mathrm{n} \cdot \Delta \mathrm{V}} .
$$

Avec les valeurs numériques suivantes : $\lambda=650 \mathrm{~nm}$, $\mathrm{n}=280$ et $\Delta \mathrm{V}=11,8 \mathrm{~V}$ (écart de tension entre 4 maximums), on obtient $d_{33}=39010^{-12} \mathrm{~m} / \mathrm{V}$, ce qui est en parfaite cohérence avec la donnée du fabricant $\left(\mathrm{d}_{33}=\right.$ $\left.40010^{-12} \mathrm{~m} / \mathrm{V}\right)$.

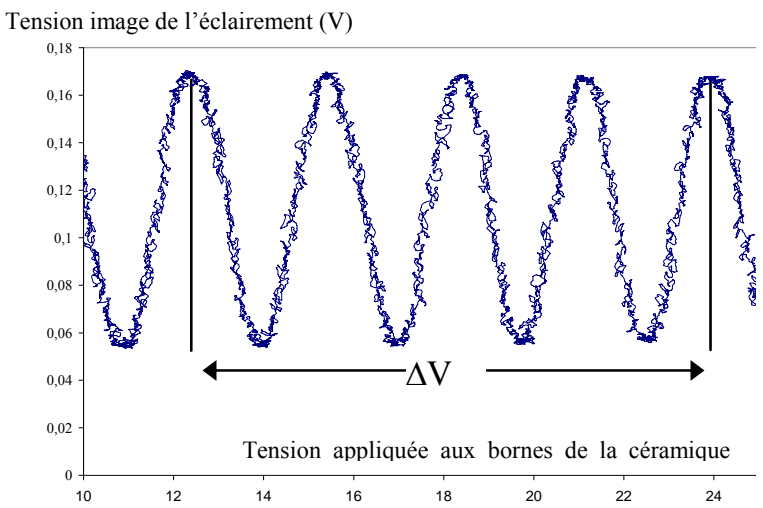

fig 14 : Évolution de l'éclairement en fonction de la tension appliquée sur la céramique

\section{CONCLUSION}

Le retour que nous avons eu sur cette manipulation de physique appliquée, après une année d'enseignement, est très positif. En effet, les étudiants découvrant les interférences lumineuses et ayant préparé cette séance parviennent à obtenir une figure d'interférences tout en comprenant les phénomènes physiques associés. Quant à ceux qui, au cours de leur scolarité, avaient déjà réglé un interféromètre, ils ont apprécié cette application concrète. De plus, cette manipulation est l'occasion de présenter des matériaux qui sont d'actualité et offrent de nouvelles perspectives.

\section{Bibiographie}

[1] B Nogarède, «Électrodynamique appliquée », Éditions Dunod 2005.

[2] J.-F Rouchon, « Enjeu des actionneurs piézoélectriques faces aux applications aéronautiques ", Journées 2004 de la section électrotechnique du club EEA.

[3] http://www.piceramic.de, céramiques utilisées.

[4] Techniques de l'ingénieur, «Mesures dimensionnelles par interférométrie laser », R1320. 